\title{
Téoros
}

Revue de recherche en tourisme

\section{Le choc européen des marchés}

\section{François Maurice}

Volume 9, numéro 3, novembre 1990

La distribution du produit touristique

URI : https://id.erudit.org/iderudit/1079887ar

DOI : https://doi.org/10.7202/1079887ar

Aller au sommaire du numéro

Éditeur(s)

Université du Québec à Montréal

ISSN

0712-8657 (imprimé)

1923-2705 (numérique)

Découvrir la revue

Citer cet article

Maurice, F. (1990). Le choc européen des marchés. Téoros, 9(3), 22-27.

https://doi.org/10.7202/1079887ar d'utilisation que vous pouvez consulter en ligne.

https://apropos.erudit.org/fr/usagers/politique-dutilisation/ 


\section{Le choc européen des marchés}

Lorsque nous avons commencé en 1972 nos recherches sur l' industrie européenne des voyages aériens à forfait, eten particulier française, celle-ci n'avait pas encore fait l'objet d'une recherche universitaire en France $^{(1)}$.

En effet, ce phénomène économique avait une existence très récente, puisqu'en 1949 . l'organisateur de voyages britannique Horizon Holidays, commercialisait, peutêtre pour la première fois dans l'histoire européenne, un voyage aérien à forfait ${ }^{(2)}$.

La croissance très rapide de cette industrie a été rendue possible par la création d'une industrie fondée essentiellement sur l'intégration d"un organisateur de voyages avec une compagnie d'affrètement, une hôtellerie de vacances et un réseau de dis tribution. "L'intégration verticale dans les activités de voyages commence dès que l'on réalise qu'il n'existe qu'un seul marché des loisirs et lorsqu'elle cherche à y contrôler suffisamment d'éléments pour réaliser des économies d'échelle et mettre sur le marché un produit intégré"(3).

Avec plus de 320 millions d'habitants, la Communauté économique européenne (C.E.E.) représente le marché le plus peuplé du monde industrialisé ( 245 millions aux États-Unis, 120 millions au Japon). Mais l'Europe reste une mosaïque de petits pays, qui ont hérité de leur histoire des caractéristiques qui les différencient.

En 1985, les habitants de la C.E.E. ont réalisé 212,8 millions de séjours de vacances, le premier marché du monde des vacances. A l'horizon 1993, ils seront 262 millions de vacanciers et en 2000, peut-être 323 millions. Une croissance aussi forte,

\footnotetext{
* Monsieur François Maurice est president de l'Association internationale des docteurs en economie du tourime. Ce texte est un large extrait de la conference quil a prononcese au Maroc a l'irvitation de M. Moulay Zine El Abdine Alacui, président de la Société marocaine de gestion höteliére SAFIR. Cette conférence dont le titre original est Analyse des constguences economiques de l'Acte unique europeen sur Findustrie europtenne des voyages a forfait fut donnee lors du Salon international du tourisme (Satour-Casablanca-Maroc) le 20 janvier 1990.
}

au rythme annuel de $+2,8 \%$, sur une période aussi longue s'explique par le fait que la consommation vacancière ne dépend plus du revenu de la période des ménages, mais d'un ensemble complexe d'anticipation éminent subjectif.

Cette analyse $s^{\prime}$ inscrit dans le cadre de deux événements majeurs:

- le premier, un fort développement de la consommation des voyages aériens à forfait;

- le second, la constitution d'un marché des vacanciers et des transports aériens européens d'ici au 1er janvier 1993.

Mise à part la concurrence des aéroports périphériques dans les régions limitrophes, les industries nationales des États européens n'ont guère connu de concurrences internationales.

Or, en 1993, la concurrence que rencontreront les compagnies aériennes européennes et les organisateurs de voyages européens sesituera au départ de chaque pays de la Communauté. L'espace commercial d'un vol affrété ne sera plus limité à un pays émetteur pour un pays récepteur, mais à plusieurs. Un organisateur devoyage pourra commercialiser un même hôtel avec un même vol affrété prenant en charge des vacanciers dans deux pays.

Or, les industries des voyages aériens à forfait de l'Europe du Nord bénéficient d"un développement considérable avec une production très concentrée, spécialisée dans le trafic bas de gamme à destination de la région Europe-Méditerranée, avec une excellente compétitivité-prix.

Àl'opposé, les marchés del'Europe du Sud ne sont pas orientés vers une production de masse, peu élaborée, mais vers une production très sophistiquée, de haut et moyen de gamme. Ce sont ces marchés qui se placent au premier rang pour les trafics à destination des régions intercontinentales.

En 1993, nous allons donc assister au choc: deces marchés. Les organisateurs de voyages de l'Europe du Nord vont être à la recherche du trafic bas de gamme de l'Europe du Sud pour compléter leur remplissage hôttelier et/ou aérien. De mềme, les organisateurs de voyage de l'Europe du Sud rechercheront le trafic haut et moyen de gamme de l'Europe du Nord.

Le réarmement industriel a déja commencé dans toute 1'Europe en vue du choc 1993. Pour décrire l'état du "champ de bataille", nous pouvons distinguer deux camps:

\section{L'Europe du Nord: une industrie parfaitement dimensionnée avec la meilleure efficacité productive}

Les organisateurs de voyages de $1^{3}$ Europe du Nord dominent la filière des produits de bas de gamme a destination de la région Europe-Méditerranée. Ces industries touristiques bénéficientd' unespécialisation dans les produits simples où la compétitivitéprix joue un rôle fondamental. L'Allemagne fédérale, la Grande-Bretagne, le Danemark, les Pays-Bas et la Belgique n'ont pas développé une spécialisation très intense de leur production, mais ces pays ont organisé leurs points forts de leurs industries touristiques en ensembles cohérents qui font leur force: organisateurs de voyages intégrant la distribution, le transport, l'hôtellerie et le réceptif.

L'existence d'une relation entre la dimension des entreprises et leur performance économique revêt une importance toute particulière dans la perspective del'émergence du grand marché unique européen.

À partir de l'étude d'un échantillon de nombreux organisateurs de voyages, nous avons cherché à déterminer la dimension "optimale" d'un organisateur de voyages, c'est-à-dire celle qui correspond à la meilleure efficacité productive. Dece point de vue, les organisateurs de voyages du "Nord" sont parfaitement dimensionnes pour le trafic bas de gamme à destination de la région Europe-Méditerranée.

Ils contrơlent en général $25 \%$ à $30 \%$ du marché national des voyages à forfait et dépassent le seuil des 1,5 millions de vacanciers. Par contre, ils sont sousdimensionnés pour le moyen et haut de gamme, en particulier à destination des régions intercontinentales. 
La compétitivité-prix des voyages aériens à forfait produits par l'Europe du Nord a permis de forts gains de parts de marché, résultant d'une production intégrée et de rendements $\mathrm{d}^{\prime} 6$ chelle croissants.

Rappelons que l'élargissement de la taille des marchés sur lesquels opèrent les organisateurs de voyages conduit généralement à un accroissement de leur propre taille. Or, $1^{\prime}$ accroissement de taille conduit à réduire les coûts de production, par l'existence de rendements d'échelle croissants, et par l'amélioration de la compétitivité.

\section{L'Europe du Sud: une industrie spécialisée dans les produits haut et moyen de gamme et dans le pôle de l'hôtellerie de vacances}

L'Europe du Sud (France, Italie, Espagne et la Suisse ${ }^{(4)}$ est spécialiste des produits moyen et haut de gamme et dispose d'une spécialisation de pôle: l'hôtellerie de vacances. Elle occupe donc une position également très défendable: les industries touristiques ont une prééminence très forte pour les produits moyen et haut de gamme, en particulier à destination des régions intercontinentales. Elle surclasse le "Nord" en voyages aériens, à forfait, à motivations spécifiques (tennis, golf, chasse, pêche, safaris, etc.) et à haute technologie (hôtels de loisirs, villages de vacances, circuits à thème, etc.). Par contre, leur spécialisation dans le bas de gamme est particulièrement faible.

Par exemple, la France bénéficie d'une spécialisation dans la production haut et moyen de gamme unique dans la CEE (mis à part le cas de la Suisse) $)^{(5)}$. Elle a développé une spécialisation pointue sur des créneaux qu'elle maîtrise parfaitement: l'hôtellerie de vacances, les produits à motivations, les voyages de stimulation etde congrès. Ceuxcidominent maintenant son tissu industriel touristique. L'Europe du Nord n'a pas développé une spécialisation aussi intense, elle se cantonne pratiquement dans une production bas de gamme ${ }^{(6)}$ et son tissu productif souffre d'une vocation de généraliste dans la production à bas prix.

Il y a donc une certaine complémentarité entre "le Nord" et "le Sud", même si les rivalités vont apparaître.

\section{La mise en place du grand marché intérieur touristique: les effets d'intégration des marchés}

La création d'un grand marché européen de vacances permettra d'augmenterl'efficacité de certaines productions bas et moyen de gamme, dans l'hypothèse de rendements d'échelle croissants. L'élargissement de la taille des marchés sur lesquels opèrent les organisateurs de voyages conduira à un accroissement de leur propre taille. Or, l'accroissement de taille conduit à réduire les coûts de production et à améliorer la compétitivité. Le renforcement de la concurrence est un ingrédient important pour la réussite du marché intérieur. La concurrence implique généralement à la fois une baisse des prix de vente et du taux de marge prix/coûts pour les organisateurs de voyages. Or, la dispersion des prix entre les pays dela la Communautéest généralement très grande: environ $30 \%$ a $45 \%$ pour les produits bas de gamme, $10 \%$ à $15 \%$ pour les produits haut et moyen de gamme. Le consommateur y sera à priori gagnant à court terme. A moyen terme, certains organisateurs de voyages dont les marges seront laminées devront disparaître en faveurdes plus productifs. Lerenforcement de laconcurrence poussera les entreprises à rechercher une meilleure efficacité productive, et à affiner constamment leur stratégie de développement. Elle conduira a la résorption des graisses organisationnelles et à des accords de coopération horizontale (entre organisateurs de voyages) ou vertical (distribution, transport).

Pour la production bas de gamme, la baisse des prix passera obligatoirement par l'accroissement de la propre taille des organisateurs de voyages. Pour le moyen et haut de gamme (les secteurs très spécialisés), les petits et moyens organisateurs de voyages continueront de coexister, car les fortes différenciations des produits permettront d'exploiter les économies d'échelle sur des niches de taille parfois petites (ex:: trafic long courrier).

Nous connaîttrons cependant des risques de pouvoirs oligopolistiques pour le trafic bas de gamme àdestination de la région EuropeMéditerranée.

\section{Concrétiser les opportunités}

La rếussite đu marché intérieur repose très largement sur la capacité des entreprises touristiques à saisir les opportunités et à les concrétisergrâce à des réactions stratégiques adequates.
Les organisateurs de voyages à forfait de l'Europe du Nord à la recherche du trafic bas de gamme de l'Europe du Sud pour compléter leur remplissage hôtelier

Le groupe allemand T.U.I. n'est présent qu'en France (Touropa) et en Autriche (Touropa). Legroupe anglais Thomson n'a aucune implantation européenne $e^{(7)}$. Le groupe danois Tuaereborg n'existe qu'en Scandinavie, au Royaume-Uni et en Allemagne. Le groupe belge Sun International $n^{4}$ est présent qu'aux PaysBas, au Royaume-Uni et en France.

L'internationalisation de ces groupes a toujours échoué pour des problèmes de transport aérien et de logistique télématique. Or, nous constatons que ces groupes ont cré des filiales dans des pays où ils pouvaient disposer d'un transport aérien à des prix relativement bas ou pour s'assurer des compléments régionaux de trafic. L'échec de l'implantation de Neckermann, en 1973, en France tient en grande partie au transport aérien.

En 1993, nous pourrions imaginer que les organisateurs de voyages de $\mathrm{l}^{\prime} E$ Europe du Nord soient tentés de trouver des compléments de remplissage aérien ou hôtelier en France, Italie et Espagne.

En effet, une grande partie des vols affrétés à destination de la Méditerranée transite via ces pays. En 1993, nous pourrions supposer que, par exemple, $T_{\text {. }} \mathrm{U}_{\text {.I. }}$ commercialise un voyage aérien à forfait à destination des Canaries au départ de Hambourg, avec une escale de complément de remplissage à Paris ou à Milan.

De plus, les transporteurs européens se dotent de filiales d'affrètement dans les pays méditerranéens de grande capacité touristique de façon à avoir des avions basés dans ces pays et desservir ainsi économiquement le pays d'origine des vacanciers.

L'implantation des compagnies d' affrètement de l'Europe du Nord en France, en Espagne etlou en Italie

- LaCompagnie Aviaco(filiale d'Iberia, Condor (filiale de Lufthansa) et Air Europe (filiale de International Leisure Group) ont crés en 1987 une filiale commune en Espagne.

- Les Compagnies Alitalia et Lufthansa étudient la création d'une filiale commune d' affrètement en Italie. 
La Compagnie Air Europe (filiale dI.L.G.) vient de créer une compagnie pan-européenne avec plusieurs transporteurs. Elle va créer une filiale en France. Elle a commandé 13 Boeing 737-400 et 26 Boeing 767-200 à cet effet, soit 39 avions... Elle a transporté 2 millions de passagers en 1987 avec des prix de $30 \%$ a $35 \%$ inférieurs à la France" "Un appareil chez Air France emploie 400 personnes, chez British Airways 300, chez Air Europe $75^{\circ}$, souligne Monsieur Harry Goodman, président d'I.L.G. ${ }^{(9)}$. En Espagne, sa filiale Air Europa dispose de 4 Boeing 737 et d'un Boeing $757^{\text {(10) }}$.

La Compagnie Air Europe a pris une participation de $49 \%$ dans le transporteur aérien régional allemand N.F.D..

- La Compagnie belge T.E.A. d'affrètements'implante en France et a commandé 17 Boeing 737-300. En janvier 1989 , elle a créé une filiale française à Lille dont $51 \%$ du capital est détenu par le Crédit du Nord et Worms. Rappelons qu'elle dispose d'une filiale en Grande-Bretagne, AirUK.

- Les groupes touristiques de l'Europe du Nord sont donc en train de créer des "hubs" à partir de leurs aéroports de départ (Londres, Bruxelles, etc.) pour concentrer le trafic d'un nombre considérable de villes de manière à contrôler le maximum de liaisons non régulières internationales à leur bénéfice exclusif. Ils créent ensuite ou utilisent des transports "feeders" avec des compagnies importantes qui satelliseront de petites compagnies qui acheminent le trafic de vacanciers vers les aéroports de départ des vols des organisateurs de voyages.

\section{Les synergies des organisateurs de voyages a forfait}

Monsieur Rudolphe Van Moerkerke, président du groupe belge Sun Intemational déclare récemment: "Je constate qu'il y a deux marchés quasiment impénétrables: le britannique, dominé par Thomson, et l'allemand sur lequel règne T.U.I., Ces deux organisateurs de voyages seront évidemment au "top niveau" en 1993. Mais je crois tout autant dans les chances de ceux qui s'associeront et créeront des synergies par delà les frontières." "(11)

Voyons les synergies européennes en cours deréalisation: les exemples del'Allemagne fédérale et de la Bielgique.

\section{a) L'Allemagne fédérale}

* International Tourist Service (I.T.S.)

Cetorganisateur de voyages dispose d'une puissance financière très importante, car c'est la filiale des grands magasins Kaufkof, Hertie, et de lachaîne de distribution GlucksReisen.

Cette société contrôle $50 \%$ du capital d'Holland International Travel (400 000 vacanciers) aux Pays-Bas depuis 1987. Elle possède également $20 \%$ du capital de Sun Parc, filiale de Sun International S.A. (Belgique) et contrôle $47 \%$ du capital de Sun International ${ }^{(12)}$.

Le trafic consolidé d'I.T.S. avec sa filiale allemande Jet Reisen atteint 1339200 vacanciers en 1988.

\section{* T.U.I.}

Cette société dispose d'un actionnariat très éparpillé. Il ne semble pas qu'elle souhaite se développer en Europe, bien qu'elle envisage au travers de Touropa France un autre développement.

Les villages de vacances Robinson gêrés par sa filiale hôtelière peuvent être commercialisés en Europe du Sud.

\section{* Neckermann und Reisen}

Le groupe a retrouvé son équilibre d'exploitation depuis 1986 et cherche à $s$ 'associer avec un organisateur de voyages français.

Les villages de vacances Aldiana gérés par sa filiale hồtelière peuvent être également vendus en Europe du Sud.

\section{* L.T.T}

Cet organisateur de voyages détenu par la compagnie d'affrètement L.T.U. souhaite acheter ou s'associer avec un organisateur de voyages français. Sa production est essentiellement du très bas de gamme.

Les organisateurs de voyages allemands disposent des moyens de financement pour contrôler très rapidement une grande part des marchés de $1^{\top}$ Europe du Sud spécialisés dans le bas de gamme.

\section{b La Belgique}

Sun International souhaite atteindre une taille critique d' 1,4 millions de vacanciers et un chiffre d'affaires de 25 milliards de francs belges a l'horizon 1995. Monsieur R. Van Moerkerke, président de Sun International affirme: "Sun International a aujourd'hui les meilleures chances de se développer dans une sorte de croissance naturelle, grầce à l’élargissement du marché européen. Je ne veux pas être le no 1 , ce serait utopique, face aux trois millions de clients du britannique Thomson. Ce que je veux, c'est atteindre 1,4 millions de clients et un chiffre d'affaires de 25 milliards de francs belges, d'ici cinq ans, ce qui nous placera parmi les cinq premiers T.O. européens." ${ }^{\prime(13)}$

En décembre 1988, Sun International décidait de prendre le contrôle de $57 \%$ du capital de Voyage Conseil, filiale du Crédit. Agricole.

Cet accord ne concerne que 60 Caisses régionales sur 84 , mais en réalité Sun International pourra diffuser sa production au travers des 4500 points de vente du Crédit Agricole.

Cette intégration verticale va permettre à Sun International de devenir l'un des quatre plus importants organisateurs de voyages français d'ici trois ans.

Cet accord, mếme s'il est très coûteux pour legroupe belge, va lui permettre d'accroittre son rôle d'organisateur de voyages "intermediaires" dans le trafic nord/sud.

En effet, dans le nord de la France, le différentiel de prix entre les voyages à forfait produits en France et en Belgique étant d'environ $15 \%$ à $20 \% \%^{(14)}$, Sunair organise déjà des départs du nord de la France avec un transfert en autocar à l'aéroport de Bruxelles. Depuis 1'hiver 1988-1989, cette politique de détoumement de trafic de l'aéroport de Lille a pris une ampleur inquiétante ${ }^{(15)}$.

Le 20 janvier 1989, l'organisateur de voyages allemand International Tourist Service (I.T.S.) prenait $47 \%$ du capital de Sun International.

Mais, cette concurrence européenne frappera particulièrement les organisateurs de voyages spécialisés dans la production de bas de gamme de l'Europe du Sud.

Effet de tailles, synergies technologiques, commerciales et du transport: cen'estqu"à ces conditions que les firmes pourront réellement tirer profit de l'Europe.

Les organisateurs de voyages à forfait de l'Europe du Sud à la recherche du trafic haut de gamme de l'Europe du Nord

Les organisateurs de voyages du "Sud" seront tentés d'accroître leur 
commercialisation dans $l^{b}$ Europe du Nord. Car ces pays n'ont pas développé une spécialisation dans le haut et moyen de gamme aussi intense que dans le "Sud".

Les chaînes hôtelières allemandes (Robinson, Aldiana, I.T.C., I.T.H.), anglaises (Holiday Club International) et belges (Sun Club) ont un développement relativement moins important que les chaînes de l'Europe du Sud, en particulier française (ex.: Club Méditerranée).

Les filières haut et moyen de gamme de $1^{\prime}$ Europe du Sud seront-elles attaquées par le Nord?

La compétitivité-prix et la compétitivité hors-prix de l'Europe du Sud

La bas niveau des prix du transport aérien affrété et la dimension optimale des organisateurs de voyages de l'Europe du Nord procurent a priori de nets avantages comparatifs à sa production.

La compétitivité-prix de la production bas degamme de l'Europe du Nord a encouragé les organisateurs de voyages de $1^{\prime}$ Europe du Nord à s'implanter dans le "Sud".

Dans les années à venir, la concurrence de l'Europe du Nord va se préciser. Elle va peut-être se jouer à coups de rachats. Nous avons vu que les organisateurs de voyages de l'Europe du Nord sont riches et très bien dimensionnées. Leur productivité compte parmi les meilleures de laC.E.E.. Dans leur pays respectif, ces groupes touristiques ont engagé une course aux parts de marché. La concentration de la production en Allemagne fédérale, Grande-Bretagne et Danemark a atteint un degré optimal.

L'existence d'une relation entre 1a dimension des entreprises et leur performance économique revêt une importance toute particulière dans la perspective del'émergence du grand marché unique européen. Car sa création permettra d'augmenter l'efficacité de certaines productions bas degamme, dans l'hypothèse de rendements d'échelle croissants.

L'Europe du Sud bénéficie d'une compétitivité hors-prix non négligeable. Le terme "compétitivité hors-prix" désigne la capacité de production d'une industrie touris tique à capter la demande des autres pays ou de la sienne propre grăce à des facteurs autres que le prix.

Or, l'Europe du Sud offre une gamme de produits répondant à la diversité de la demande vacancière. Il convient donc qué les Êtats de l'Europe du Sud se positionnent sur des produits dont la demande croit plus vite, pour créer des positions dominantes grâce à une avance technologique et à la qualité de ses produits, se protégeant ainsi de la concurrence par les prix. L' hôtellerie de vacances devrait jouer un rôle fondamental, au travers des hôtels loisirs et villages de vacances.

\section{Les risques de pouvoirs oligopolistiques de l'Europe du Nord}

Il semble que l'Europe du Nord jouera une concurrence maximale pour le trafic bas de gamme à destination de la région EuropeMéditerranée. Le "Sud" n'aura guère de moyens pour résister.

Pour la filière moyen et haut de gamme, le "Sud" devrait avoir une bonne capacité de résistanceet même disposerait de lacapacité industrielle d'envahir le "Nord".

Mais il y aura une limite très importante aux échanges. Cette entrave, aucune loi économique peut lasupprimer: ladifférence de culture entre les Latins et les AngloSaxons.

Cette différence de culture constitue un véritable rempart de protection du "Sud" contre les tendances impérialistes du "Nord".

Rappelons que dans l' hôtellerie de vacances, et encore plus dans les circuits, il est difficile de faire cohabiter ensemble ces deux cultures en particulier dans les produits bas de gamme (ex:: villages de vacances).

Si cette hypothèse se vérifie, la division européenne del'organisation des voyages à forfait auraitses limites. L'Europe du Nord dominerait en effet la production européenne du bas de gamme, mais elle laisserait le champ libre à l'Europe du Sud pour la production hautet moyen degamme.

\section{Le développement des marchés européens}

L'accroissement de la demande vacancière et l'interpénétration des économies touristiques européennes qui caractérise les sept dernières années de ce siècle prennent leur origine dans une spécialisation mutuellementavantageuse (le bas de gamme d'une part et le haut et moyen de gamme d'autre part). Mais le jeu n'est qu'en partie coopératif et les gains ne sont pas équitablement répartis à l'avance. Voyons quels seront les grands émetteurs de vacanciers dans la C.E.E. à l'horizon 1993 et 2000 .

La demande vacancière au départ de la C.E.E.

TABLEAU 1

La demande vacancière au départ de la C.E.E.

Unité = million de vacanciers

\begin{tabular}{lrrr}
\hline & 1985 & 1993 & 2000 \\
\hline National & 149,0 & 174,6 & 201,0 \\
Étranger & 63,8 & 87,4 & 123,0 \\
\hline C.E.E. & 212,8 & 262,0 & 324,0
\end{tabular}

Sources: 1985 - C.E.E.

Prévisions 1993 et 2000

- F. Maurice

Compte tenu du développement de la consommation finale des ménages dans la C.E.E.et de lacroiss ance du pouvoird'achat touris tique, nous prévoyons pour l'horizon 2000:

- une croissance de $+2 \%$ en moyenne annuelle des vacances effectuées sur le territoire national entre 1985 et 2000 .

- une croissance de $+4,5 \%$ en moyenne annuelle des vacances effectuées à l'étranger (autres États de la C.E.E. ou autres pays).

A l'horizon 2000, les habitants ${ }^{16}$ de la C.E.E. devraient réaliser environ 324 millions deséjours devacanciers. Ceserait le premier marché des vacances du monde $e^{(1 \bar{T})}$

Voyons les principales destinations du trafic à destination de l'étranger.

A l'horizon 2000 , le trafic à destination de l'étranger devrait atteindre 123 millions, soit une croissance moyenne de $+4,5 \%$ par an. 
TABLEAU 2

Les principales destinations des vacanciers à l'étranger au départ des États de la C.E.E.

Unité $=$ million de vacanciers

Destination

$\begin{array}{lll}1985 & 1993 \quad 2000\end{array}$

Région Europe-Méditerranée

$$
59,6 \quad 82,5 \quad 117,0
$$

Régions intercontinentales

\begin{tabular}{rrrr} 
& 4,2 & 4,9 & 6,0 \\
\hline Étranger & & & \\
& 63,8 & 87,4 & 123,0 \\
\hline
\end{tabular}

Sources: 1985 - C.E.E.

Prévisions 1993 et 2000

- F. Maurice

À destination de l'étranger, les vacanciers des États de laC.E.E. partiraient en vacances essentiellement dans la région EuropeMéditerranée avec une croissance moyenne de $+4,6 \%$ entre 1985 et 2000 .

De 1985 à 1993, nous estimons que le trafic progresserait de $+4,1 \%$ en moyenne et de 1993 a 2000 de $+5,1 \%$. La réalisation du marché unique de 1993 devrait accroître sensiblement la croissance.

A destination des régions intercontinentales, le trafic $s^{5}$ accroittrait au rythme de $+2,4 \%$ entre 1985 et 1990 . De 1985 a 1993 , le marché augmenterait d'une manière lente d'environ $+2 \%$. De 1993 à 2000 , le taux serait de $+3 \%$. La constitution du marché commun des vacances constituerait donc également un formidable vecteur de développement de la demande.
L'Acte unique européen va accélérer la croissance de la demande vacancière, car la baisse du coût du transport aérien, notamment, va permettre cette multiplication des départs. Voyons le developpement de la demande vacancière dans les principaux États de la C.E.E.

\section{Au départ d'Allemagne fédérale}

\section{TABLEAU 3}

La demande vacancière au départ d'Allemagne fédérale

Unitế: million de vacanciers

\begin{tabular}{ccc}
\hline \multicolumn{1}{c}{1985} & 1993 & 2000 \\
\hline $\begin{array}{c}\text { Vacances effectuées } \\
\text { sur le territoire national } \\
14,4\end{array}$ & 15,2 & 16,7 \\
Vacances effectuées & & \\
à l'étranger & & \\
\hline 26,4 & 32,2 & 38,9 \\
\hline $\begin{array}{c}\text { Total des vacances } \\
\text { dés Allemands } \\
40,8\end{array}$ & 47,4 & 55,6 \\
\hline
\end{tabular}

Source: 1985 - S.T.F.T.

Prévisions 1993 et 2000 - F. Maurice

La demande vacancière connaîtrait une croissance d'environ $+1,9 \%$ de 1985 à 1993 et d'environ $+2,3 \%$ de 1993 à 2000 .

L'Allemagne resterait le premier pays émetteur de vacanciers, à destination de l'étranger, de la C.E.E..

\section{Au départ de la Grande-Bretagne}

\section{TABLEAU 4}

La demande vacancière au départ de la Grande-Bretagne

Unité: million de vacanciers

\begin{tabular}{ccc}
\hline \multicolumn{1}{c}{1985} & 1993 & 2000 \\
\hline $\begin{array}{c}\text { Vacances effectuées } \\
\text { sur le territoire national } \\
33,0\end{array}$ & 29,1 & 24,4 \\
$\begin{array}{c}\text { Vacances effectuées } \\
\text { à l'étranger } 15,75\end{array}$ & 25,3 & 38,0 \\
\hline $\begin{array}{c}\text { Total des vacances } \\
\text { des Britanniques } \\
48,75\end{array}$ & 54,4 & 62,4 \\
\hline
\end{tabular}

Source: British Tourism Survey Prévisions 1993 et 2000 - F. Maurice

De 1985 à 1993, la demande progresserait à un rythme d'environ de $+1,4 \%$ mais à destination de l'étranger, elle atteindrait $+6,1$.

Entre 1993 et 2000 , le taux de croissance s"accélérerait sensiblement à un rythme de $+2,0 \%$, età destination de l'étranger $+6,0 \%$.

A l'horizon 2000, la Grande-Bretagne devrait se situer au niveau de l'Allemagne fédérale, pour la demande vacancière, à destination de l'étranger.

\section{Au dêpart de la France}

\section{TABLEAU 5}

La demande vacancière au départ de la France

Unité: million de vacanciers

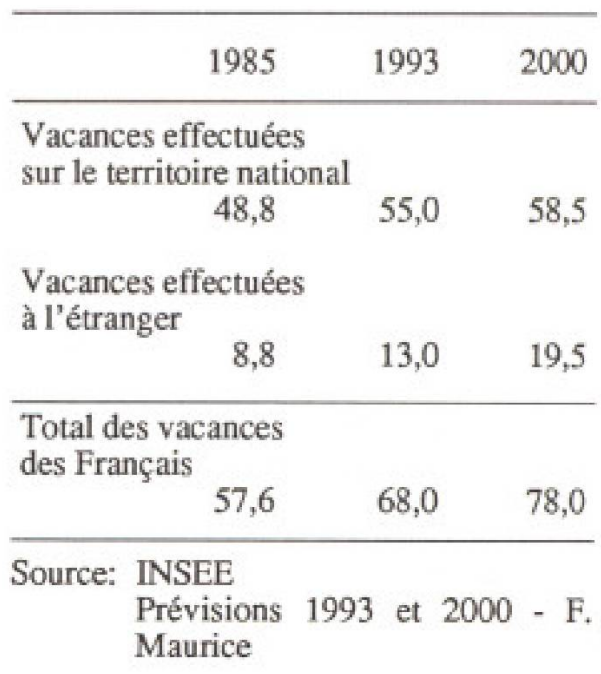


De 1985 à 1993, la demande vacancière devrait s'accroittre à un rythme de $2,1 \%$. Entre 1993 et 2000 , le taux devrait se stabiliser.

La France devrait être le plus important émetteur de vacanciers de la $\mathrm{C} . \mathrm{E} . \mathrm{E}$.

Les yacanciers à destination de l'étranger devrait progresser de $+5 \%$ entre 1985 et 1993 et $+6 \%$ entre 1993 et 2000 .

La France se classerait au troisième rang de la C.E.E. pour les vacances effectuées à l'étranger.

Or, $65 \%$ du trafic international de vacanciers de l'Allemagne fédérale et du RoyaumeUni transiterait par la France, par voie terrestre ou aérienne. Nous pouvons done imaginer que les organisateurs de voyages allemands ou britanniques développeraient leur trafic de voyages aériens à forfait à destination de la France et utiliseraient les aéroports français comme escales intermédiaires où ils débarqueraient du trafic à destination de France et embarqueraient du trafic à destination des régions intercontinentales et de la région EuropeMéditerranée.

\section{Horizon 1993/2000 - L'industrie vacancière devient une industrie lourde}

La demande vacancière dans la C.E.E. devrait connaitre une croissance très forte dans les quinze prochaines années. Elle devrait ettre dominée par trois pays: l'Allemagne fédérale, la Grande-Bretagne et la France. Ceux-cicontrôleraient environ $60 \%$ de la demande vacancière. Mais de nouveaux pays émetteursde vacanciers vont apparaittre: l'Espagne et l'Italie.

En France, en 1987, l'ensemble des activités liẹes au tourisme réalisait un chiffre d'affaires de l'ordre de 495 milliards de francs, soit un montant analogue à celui de l'ensemble des industries du bâtiment et des travaux publics. La consommation touristique intérieure représente déjà $7,4 \%$ du produit intérieur brut français.

Lademande vacancièreeuropéenne devrait devenir l'un des principaux instruments d'échange dans le dialogue Nord-Sud préconisé par la C.E.E.. Cette industrie touristique va devenir" "LE PÉTROLE BLEU DE LA C.E.E.".
Notes explicatives

(1) These de doctorat, L'Industrie europesenne dos vovages adrians à forfait, Faculté d’Ecconomie appliquese, Centre des hautes etudes touristiques. Université d'Aix-Marseille III.

(2) Horizon Holidays a été fondé en 1949 a Londres.

(3) HUDSON, E., L'intégration varticale dans I'industrie des voyages et des lolsirs, Paris, Institut du transport aerien, 1972.

(4) La Suisse ne fait pas partie de la C.E.E. mais peut tre classese dans ce groupe. car i'industrie touristique suisse est deja implantée dans la C.E.E.

(5) Mis a part quelques exceptions, notamment en Allomagne fidobrale.

(6) Sauf des filiales chargtes du receptif en Espagne, France, etc.

(7) Le Monde, 12.03.89.

(B) Tourhabdo, 1002.88

19) Air Europe a fait voler 5000 heures ses avions en 1986 .

(10) L'Écho Touristique, 0601.89

(11) Le 20.01.89. I.T.S prend une participation de $47 \%$ (2 milliards de francs belges) dans le capital de Sun International ( 5 milliards de francs belges). Or, Sun International contrôle $57 \%$ du capital du distributeur et organisateur de wovages français Voyage Conseil.

(12) L'Écho Teuristique, 06.01.89.

(13) Neckermann Belgique commercialise sa production dans le Nord de la France depuis l'hiver 1988-1989.

(14) Trafic bas de gamme a destination de la réglon Europe-Méditerranée.

(15) De plus de 15 ans.

(16) En 1993, la part de l'avion serait d'environ $17 \%$ et en 2000 emviron $20 \%$, contre $13 \%$ en 1985 . 